\title{
Unparticle phase effects
}

\author{
Chuan-Hung Chen ${ }^{1,2 *}$ and Chao-Qiang Geng ${ }^{3,4 \dagger}$ \\ ${ }^{1}$ Department of Physics, National Cheng-Kung University, Tainan 701, Taiwan \\ ${ }^{2}$ National Center for Theoretical Sciences, Taiwan \\ ${ }^{3}$ Department of Physics, National Tsing-Hua University, Hsinchu 300, Taiwan \\ ${ }^{4}$ Theory Group, TRIUMF, 4004 Wesbrook Mall, \\ Vancouver, B.C V6T 2A3, Canada
}

(Dated: October 27, 2018)

\begin{abstract}
Unparticles proposed by Georgi carry CP conserving phases in their propagators. We demonstrate that these peculiar phases have an important impact on $\mathrm{CP}$ violation. Without including the strong QCD phases, we study the unparticle phase effects on the direct CP asymmetries in the exclusive decays of $\bar{B}_{d} \rightarrow \pi^{+} \pi^{-}$and $B \rightarrow \pi K$, in which the flavor changing neutral currents are forbidden at tree level but induced by one-loop diagrams. Interesting and consistent results comparing to the data are obtained. In addition, we find that unparticles will significantly enhance the differential branching ratio of $b \rightarrow s \ell^{+} \ell^{-}$at the small invariant mass of $\ell^{+} \ell^{-}$. The forward-backward asymmetries for $b \rightarrow s \ell^{+} \ell^{-}$due to unparticles are also explored.
\end{abstract}

\footnotetext{
* Email: physchen@mail.ncku.edu.tw

$\dagger$ Email: geng@phys.nthu.edu.tw
} 


\section{INTRODUCTION}

In Refs. [1, 2] Georgi has suggested that a scale invariant sector with a non-trivial IR fixed point decoupled at a large scale is associated with unparticles, which could couple to the standard model (SM) particles at the TeV scale. Consequently, the unparticle physics phenomenology have been extensively explored in Refs. [1, 2, 3, 4, 5, 6, 7, 8, 9, 10, 11, 12, 13, 14, 15, 16, 17, 18, 19, 20, 21]. Moreover, Georgi in Ref. [2] has pointed out that the unparticle propagators in the time-like region are associated with some peculiar CP conserving phases depending on the non-integral number of the scale dimension $d_{\mathcal{U}}$. He has shown that these phases can induce some unusual CP conserving interference effects between the time-like unparticle exchange amplitudes and the SM amplitudes in $e^{+} e^{-} \rightarrow \mu^{+} \mu^{-}$. The effect of the virtual unparticle propagation has also been noticed in Ref. [3].

Recently, in Ref. [5] we have demonstrated that the peculiar CP conserving phases in the unparticle propagators can also play very important roles on $\mathrm{CP}$ violation. We have explicitly examined the phase effects on the direct CP asymmetries (CPAs) in $B_{d} \rightarrow \pi^{-} \pi^{+}$ and $B_{d} \rightarrow \ell^{-} \ell^{+}$decays based on operators with fermion flavor changing neutral currents (FCNCs) at tree level. We have found that the direct CPAs in both decays could be large. In this paper, we will examine the unparticle phase effects on $\mathrm{CP}$ violation with only flavor conserving operators at tree level. Specifically, we only consider those effects in which FCNCs are forbidden at tree level like the SM but they can be generated by one-loop diagrams including the penguin unparticle ones.

It is well known that in a decay process the direct CPA $\left(\mathcal{A}_{C P}\right)$ depends on two types of phases, called weak $(\delta)$ and strong $(\phi)$ phases which are CP violating and conserving, respectively. In particular, one has that

$$
\mathcal{A}_{C P} \propto \sin \delta \sin \phi
$$

Clearly, to have a sizable value of $\mathcal{A}_{C P}$, both phases have to be nonzero and large. In the SM, the weak $\mathrm{CP}$ violating phase is the unique phase in the $3 \times 3$ Cabibbo-Kobayashi-Maskawa (CKM) quark mixing matrix [22], which has been fixed by experiments [23, 24]. The CP conserving strong phase is process dependent, which is normally hard to be determined due to hadronic uncertainties. Since the unparticle phases appear in the propagators and conserve $\mathrm{CP}$, it is interesting to speculate that these phases could act as the strong phases in some physical processes [5]. To explore this possibility, we will concentrate on B decays as 
there are many experimental CP violating phenomena [24] from the current B factories as well as future super-B facilities. In particular, we will investigate direct CPAs in the decays of $B \rightarrow \pi \pi$ and $B \rightarrow \pi K$. It is clear that our study can be extended to other processes such as $K$ and $D$ decays.

The paper is organized as followed. In Sec. II, we present FCNCs which are induced by the unparticle penguin diagrams. We show the unparticle effects on charmless nonleptonic and semileptonic B decays in Sec. III. We give our numerical analysis in Sec. IV and conclude our results in Sec. V.

\section{FLAVOR CHANGING NEUTRAL CURRENTS INDUCED BY UNPARTICLE PENGUIN DIAGRAMS}

To study the low energy effects of unparticle physics, for simplicity, we assume that unparticles only couple to the flavor conserving fermion currents, described by [1, 2]

$$
\frac{1}{\Lambda_{\mathcal{U}}^{d_{\mathcal{U}}-1}} \bar{f} \gamma_{\mu}\left(C_{L}^{\mathrm{f}} P_{L}+C_{R}^{\mathrm{f}} P_{R}\right) f O_{\mathcal{U}}^{\mu}
$$

where $P_{L(R)}=\left(1 \mp \gamma_{5}\right) / 2$ and $O_{\mathcal{U}}^{\mu}$ is the vector unparticle operator. Clearly, at tree level, the fermion flavor is conserved. Similar to the SM, FCNCs such as $f \rightarrow f^{\prime} \mathcal{U}$ can be induced by the charged weak currents at one-loop level. Due to the CKM mixing matrix element $V_{t b} \approx 1$ and the heavy top-quark enhancement, we will concentrate on $B$ decays to illustrate some important physics phenomena involving unparticles. Our discussions can be straightforwardly generalized to $K$ and $D$ decays. The leading effective interaction for the

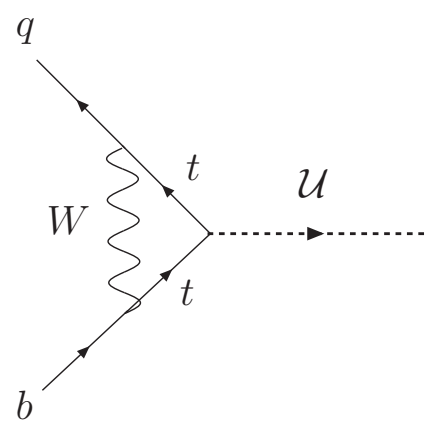

FIG. 1: Feynman diagram for $b \rightarrow q \mathcal{U}$.

flavor changing transition of $b \rightarrow q$ is induced by the unparticle penguin diagram shown in 
Fig. 1, which leads to

$$
\mathcal{L}_{\mathcal{U}}=\frac{g^{2}}{\Lambda_{\mathcal{U}}^{d_{\mathcal{U}}-1}} V_{t b} V_{t q}^{*} C_{L}^{q b} \bar{q} \gamma_{\mu} P_{L} b O_{\mathcal{U}}^{\mu}
$$

where

$$
\begin{aligned}
C_{L}^{q b} & =\frac{1}{(4 \pi)^{2}} I\left(x_{t}\right), \\
I\left(x_{t}\right) & =\frac{x_{t}\left(2 C_{R}^{t}+C_{L}^{t} x_{t}\right)}{2\left(1-x_{t}\right)^{2}}\left(-1+x_{t}-\ln x_{t}\right),
\end{aligned}
$$

with $x_{t}=m_{t}^{2} / m_{W}^{2}$. Here, we have adopted the Feynman-'t Hooft gauge and the contributions from the charged Goldstone boson have been included. For simplicity, in the following analysis, we will set $C_{R}^{t} \approx C_{L}^{t}$.

To obtain the unparticle-mediated effects, we need to know the unparticle propagator, which is given by [1, 2]

$$
\int d^{4} x e^{i p \cdot x}\left\langle 0\left|T\left(O_{\mathcal{U}}^{\mu}(x) O_{\mathcal{U}}^{\nu}(0)\right)\right| 0\right\rangle=i \Delta_{\mathcal{U}}\left(p^{2}\right) e^{-i \phi_{\mathcal{U}}}
$$

where

$$
\begin{aligned}
\Delta_{\mathcal{U}}\left(p^{2}\right) & =\frac{A_{d_{\mathcal{U}}}}{2 \sin \left(d_{\mathcal{U}} \pi\right)} \frac{-g^{\mu \nu}+p^{\mu} p^{\nu} / p^{2}}{\left(p^{2}+i \epsilon\right)^{2-d_{\mathcal{U}}}} \\
\phi_{\mathcal{U}} & =\left(d_{\mathcal{U}}-2\right) \pi
\end{aligned}
$$

with

$$
A_{d_{\mathcal{U}}}=\frac{16 \pi^{5 / 2}}{(2 \pi)^{2 d_{\mathcal{U}}}} \frac{\Gamma\left(d_{\mathcal{U}}+1 / 2\right)}{\Gamma\left(d_{\mathcal{U}}-1\right) \Gamma\left(2 d_{\mathcal{U}}\right)} .
$$

Note that in Eq. (5) the phase factor arises from $(-1)^{d_{\mathcal{U}}-2}=e^{-i \pi\left(d_{\mathcal{U}}-2\right)}$ and the vector operator is assumed to satisfy the transverse condition $\partial_{\mu} O_{\mathcal{U}}^{\mu}=0$. In terms of the effective interaction in Eq. (3) and the unparticle propagator in Eq. (5), the effective Hamiltonian for $b \rightarrow q f \bar{f}$ can be written as

$$
\mathcal{H}_{\mathcal{U}}=-\frac{G_{F}}{\sqrt{2}} V_{t b} V_{t q}^{*} \tilde{\Delta}_{\mathcal{U}}\left(p^{2}\right) e^{-i \phi_{\mathcal{U}}} \bar{q} \gamma_{\mu} P_{L} b \bar{f} \gamma^{\mu}\left(C_{L}^{\mathrm{f}} P_{L}+C_{R}^{\mathrm{f}} P_{R}\right) f
$$

where $G_{F}=8 \sqrt{2} g^{2} / m_{W}^{2}$ is the Fermi constant and

$$
\tilde{\Delta}_{\mathcal{U}}\left(p^{2}\right)=8 C_{L}^{q b} \frac{A_{d_{\mathcal{U}}}}{2 \sin d_{\mathcal{U}} \pi} \frac{m_{W}^{2}}{p^{2}}\left(\frac{p^{2}}{\Lambda_{\mathcal{U}}^{2}}\right)^{d_{\mathcal{U}}-1} .
$$

We note that $f$ can be neutrinos or charged leptons or quarks. We remark that replacing $b$ by $s$ in the effective Hamiltonian of Eq. (8), we may study $s \rightarrow q f \bar{f}$ decays. 


\section{CHARMLESS NONLEPTONIC AND SEMILEPTONIC B DECAYS}

For the nonleptonic decays of $b \rightarrow q q^{\prime \prime} \bar{q}^{\prime \prime}$, we start with the explicit expression of the effective Hamiltonian in the SM [25]

$$
H_{\mathrm{eff}}=\frac{G_{F}}{\sqrt{2}} \sum_{q^{\prime}=u, c} \xi_{q^{\prime}}^{q}\left[C_{1}(\mu) O_{1}^{(q)}(\mu)+C_{2}(\mu) O_{2}^{(q)}(\mu)+\sum_{i=3}^{10} C_{i}(\mu) O_{i}(\mu)\right],
$$

where $\xi_{q^{\prime}}^{q}=V_{q^{\prime} b} V_{q^{\prime} q}^{*}$ denotes the product of the CKM matrix elements and the operators $O_{1}-O_{10}$ are defined by

$$
\begin{aligned}
& O_{1}^{(q)}=\left(\bar{q}_{\alpha} q_{\beta}^{\prime}\right)_{V-A}\left(\overline{q^{\prime}}{ }_{\beta} b_{\alpha}\right)_{V-A}, \quad O_{2}^{(q)}=\left(\bar{q}_{\alpha} q_{\alpha}^{\prime}\right)_{V-A}\left(\overline{q^{\prime}}{ }_{\beta} b_{\beta}\right)_{V-A}, \\
& O_{3}=\left(\bar{q}_{\alpha} b_{\alpha}\right)_{V-A} \sum_{q^{\prime \prime}}\left(\overline{q^{\prime \prime}}{ }_{\beta} q_{\beta}^{\prime \prime}\right)_{V-A}, \quad O_{4}=\left(\bar{q}_{\alpha} b_{\beta}\right)_{V-A} \sum_{q^{\prime \prime}}\left(\overline{q^{\prime \prime}}{ }_{\beta} q_{\alpha}^{\prime \prime}\right)_{V-A}, \\
& O_{5}=\left(\bar{q}_{\alpha} b_{\alpha}\right)_{V-A} \sum_{q^{\prime \prime}}\left(\overline{q^{\prime \prime}}{ }_{\beta} q_{\beta}^{\prime \prime}\right)_{V+A}, \quad O_{6}=\left(\bar{q}_{\alpha} b_{\beta}\right)_{V-A} \sum_{q^{\prime \prime}}\left(\overline{q^{\prime \prime}}{ }_{\beta} q_{\alpha}^{\prime \prime}\right)_{V+A}, \\
& O_{7}=\frac{3}{2}\left(\bar{q}_{\alpha} b_{\alpha}\right)_{V-A} \sum_{q^{\prime \prime}} e_{q^{\prime \prime}}\left(\overline{q^{\prime \prime}}{ }_{\beta} q_{\beta}^{\prime \prime}\right)_{V+A}, \quad O_{8}=\frac{3}{2}\left(\bar{q}_{\alpha} b_{\beta}\right)_{V-A} \sum_{q^{\prime \prime}} e_{q^{\prime \prime}}\left(\overline{q^{\prime \prime}}{ }_{\beta} q_{\alpha}^{\prime \prime}\right)_{V+A}, \\
& O_{9}=\frac{3}{2}\left(\bar{q}_{\alpha} b_{\alpha}\right)_{V-A} \sum_{q^{\prime \prime}} e_{q^{\prime \prime}}\left(\overline{q^{\prime \prime}}{ }_{\beta} q_{\beta}^{\prime \prime}\right)_{V-A}, \quad O_{10}=\frac{3}{2}\left(\bar{q}_{\alpha} b_{\beta}\right)_{V-A} \sum_{q^{\prime \prime}} e_{q^{\prime \prime}}\left(\overline{q^{\prime \prime}}{ }_{\beta} q_{\alpha}^{\prime \prime}\right)_{V-A},
\end{aligned}
$$

with $\alpha$ and $\beta$ being the color indices, $C_{1}-C_{10}$ the Wilson coefficients (WCs), $e_{q^{\prime \prime}}$ the electric charge of $q^{\prime \prime}$ and $\left(\bar{q}^{\prime \prime} q\right)_{V \pm A}=\bar{q}^{\prime \prime} \gamma^{\mu}\left(1 \pm \gamma_{5}\right) q^{\prime \prime}$. In Eq. (10), $O_{1}-O_{2}$ are from the tree level

of weak interactions, $\mathrm{O}_{3}-\mathrm{O}_{6}$ are the so-called gluon penguin operators and $\mathrm{O}_{7}-\mathrm{O}_{10}$ are the electroweak penguin operators. Using the unitarity condition, the CKM matrix elements for the penguin operators $O_{3}-O_{10}$ can also be related by

$$
\xi_{u}^{q}+\xi_{c}^{q}=-\xi_{t}^{q}
$$

Comparing to Eq. (8), we clearly see that the structures of four-Fermi interactions with unparticle contributions are the same as those of $O_{3}$ and $O_{5}$. Consequently, we can easily get the unparticle contributions by replacing $C_{3}$ and $C_{5}$ in the SM with

$$
C_{3}^{q \mathcal{U}}\left(p^{2}\right)=C_{3}+\frac{1}{4} \tilde{\Delta}_{\mathcal{U}}\left(p^{2}\right) C_{L}^{q} e^{-i \phi_{\mathcal{U}}}, \quad C_{5}^{q \mathcal{U}}\left(p^{2}\right)=C_{5}+\frac{1}{4} \tilde{\Delta}_{\mathcal{U}}\left(p^{2}\right) C_{R}^{q} e^{-i \phi_{\mathcal{U}}} .
$$

Accordingly, the associated effective WCs could be classified and re-expressed to be more 
useful forms by

$$
\begin{aligned}
& a_{1}=C_{2}+\frac{C_{1}}{N_{c}}, \quad a_{2}=C_{1}+\frac{C_{2}}{N_{c}}, \\
& a_{3}^{q \mathcal{U}}=C_{3}^{q \mathcal{U}}+\frac{C_{4}}{N_{c}}+\frac{3}{2} e_{q}\left(C_{9}+\frac{C_{10}}{N_{c}}\right), \quad a_{4}^{q \mathcal{U}}=C_{4}+\frac{C_{3}^{q \mathcal{U}}}{N_{c}}+\frac{3}{2} e_{q}\left(C_{10}+\frac{C_{9}}{N_{c}}\right), \\
& a_{5}^{q \mathcal{U}}=C_{5}^{q \mathcal{U}}+\frac{C_{6}}{N_{c}}+\frac{3}{2} e_{q}\left(C_{7}+\frac{C_{8}}{N_{c}}\right), \quad a_{6}^{q \mathcal{U}}=C_{6}+\frac{C_{5}^{q \mathcal{U}}}{N_{c}}+\frac{3}{2} e_{q}\left(C_{8}+\frac{C_{7}}{N_{c}}\right)
\end{aligned}
$$

where $N_{c}=3$ is the number of color.

\section{A. $B \rightarrow \pi \pi$ decays}

In this section, we are going to study the decays of $B \rightarrow \pi \pi$ dictated by $b \rightarrow d q \bar{q}$. Using the effective operators displayed in Eqs. (11) and (14), it is easy to see that the decays are tree dominated. However, it is clear that the unparticle effects on $B^{-} \rightarrow \pi^{-} \pi^{0}$ could be neglected if $C_{L(R)}^{d} \approx C_{L(R)}^{u}$ as the effects are always related to $-\left(a_{3}^{u \mathcal{U}}-a_{3}^{d \mathcal{U}}\right)+\left(a_{5}^{u \mathcal{U}}-a_{5}^{d \mathcal{U}}\right)$, where the minus sign inside brackets is from the pion flavor wave function, $\left|\pi^{0}\right\rangle=(\bar{u} u-\bar{d} d) / \sqrt{2}$, and the other one outside the brackets is due to the pseudoscalar decay constant, defined by $\left\langle P(p)\left|\bar{f}^{\prime} \gamma^{\mu}\left(1 \pm \gamma_{5}\right) f\right| 0\right\rangle= \pm i f_{P} p^{\mu}$. On the other hand, as no significant CPA for $B^{-} \rightarrow \pi^{-} \pi^{0}$ is found based on the current experimental world average, it should be a good scenario to take $C_{L(R)}^{d} \approx C_{L(R)}^{u}$. Unfortunately, since the unparticle contributions arise at one-loop level, we don't expect that we can solve the problem of the large branching ratio (BR) on $B_{d} \rightarrow \pi^{0} \pi^{0}$, in which the tree contribution plays a dominant role. Hence, we will concentrate on the CPA of $B_{d} \rightarrow \pi^{+} \pi^{-}$.

It is known that the penguin effects on $B_{d} \rightarrow \pi^{-} \pi^{+}$are significant even though the decay is tree dominated, In order to generate strong phases for the CPA, in the SM the annihilation topology from $O_{6} \propto(V-A) \otimes(V+A)$ plays a very important role. With the Fierz transformation, since the corresponding QCD effects involve the timelike form factor denoted by $\left\langle\pi \pi\left|\bar{q}^{\prime}\left(1+\gamma_{5}\right) q\right| 0\right\rangle$, the theoretical calculations are very uncertain. For instance, with the QCD factorization [26], to cure divergences one needs to introduce free parameters to parametrize the corresponding form factors. With the perturbative QCD approach [27], although singularities could be removed by transverse degrees of freedom, the dominant dynamical scale is close to the nonperturbative scale which is around $1 \mathrm{GeV}$ [28]. Using the soft-collinear effective theory, it is found that at the lowest order in $\alpha_{s}$, the annihilation 
contributions are real [29]. In addition, these timelike form factors are all power suppressed in $m_{P} / m_{B}$ with $m_{P}$ being the mass of the light pseudoscalar [30]. Hence, it still needs to make lots of efforts to fix the strong phases induced by the QCD effects.

As stated before, the CP-conserved phase in the unparticle propagator could provide a kind of strong phase needed for the CPA [5]. It has been realized that the phase could contribute to the CPA of $B_{d} \rightarrow \pi^{-} \pi^{+}$with tree allowed FCNCs [5]. In this study, we take the fermion flavor conservation at tree level like the SM. To examine the influence of the unparticle phase alone, we will neglect the uncertain strong phases induced by QCD interactions. By following the effective Hamiltonian in Eq. (10), we present the decay topologies in Fig. 2 where (a) $[(\mathrm{b})]$ denotes the tree (loop) effects. Since $B_{d} \rightarrow \pi^{-} \pi^{+}$is a color-allowed process,

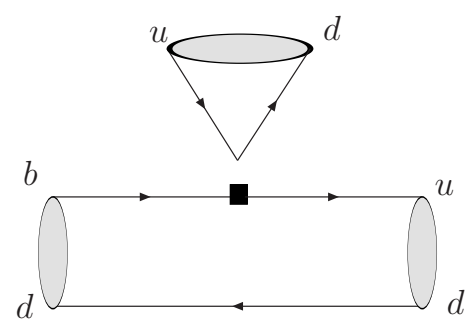

(a)

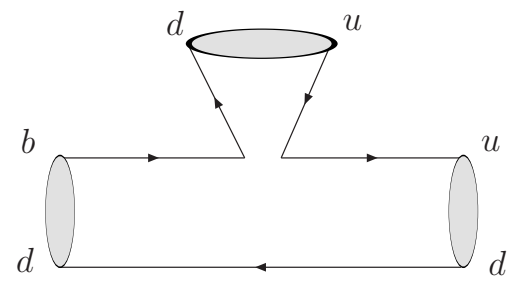

(b)

FIG. 2: Flavor diagrams from (a) tree and (b) penguin for $B_{d} \rightarrow \pi^{-} \pi^{+}$decay, where the square symbol denotes the weak vertex.

the factorization assumption is good enough to estimate the transition matrix elements. Consequently, the decay amplitude for $\bar{B}_{d} \rightarrow \pi^{+} \pi^{-}$is given by

$$
\mathcal{M}_{\pi^{+} \pi^{-}}^{\mathcal{U}}=\frac{G_{F}}{\sqrt{2}} f_{\pi} m_{B}^{2} F_{0}^{B \pi}\left(m_{\pi}^{2}\right)\left[-V_{t b} V_{t d}^{*}\left(a_{4}^{u \mathcal{U}}+2 r_{\pi} a_{6}^{u \mathcal{U}}\right)+V_{u b} V_{u d}^{*} a_{1}\right]
$$

where the form factor $F_{0}^{B \pi}$ is defined by

$$
\left\langle\pi(p)\left|\bar{u} \gamma_{\mu} b\right| \bar{B}\left(p_{B}\right)\right\rangle=\left[\left(p_{B}+p\right)_{\mu}-\frac{m_{B}^{2}}{q^{2}} q_{\mu}\right] F_{1}^{B \pi}\left(q^{2}\right)+\frac{m_{B}^{2}}{q^{2}} q_{\mu} F_{0}^{B \pi}\left(q^{2}\right)
$$

with $q=p_{B}-p$ and $r_{\pi}=m_{\pi}^{0} / m_{B}$ associated with $\left\langle\pi\left|\bar{d} \gamma_{5} u\right| 0\right\rangle=i f_{\pi} m_{\pi}^{0}$. For the light meson production in $B$ decays, we take $F^{B \pi}\left(m_{\pi}^{2}\right) \approx F_{0}^{B \pi}(0)$. Hereafter, we will use $F_{0}^{B \pi}$ instead of $F_{0}^{B \pi}(0)$. As a result, the BR and CPA could be obtained by

$$
\begin{aligned}
\mathcal{B}\left(\bar{B}_{d} \rightarrow \pi^{+} \pi^{-}\right) & =\frac{\tau_{B_{d}}}{16 \pi m_{B}}\left|\mathcal{M}_{\pi^{+} \pi^{-}}^{\mathcal{U}}\right|^{2} \\
\mathcal{A}_{C P} & =\frac{\overline{\mathcal{B}}\left(\bar{B}_{d} \rightarrow \pi^{+} \pi^{-}\right)-\mathcal{B}\left(B_{d} \rightarrow \pi^{-} \pi^{+}\right)}{\overline{\mathcal{B}}\left(\bar{B}_{d} \rightarrow \pi^{+} \pi^{-}\right)+\mathcal{B}\left(B_{d} \rightarrow \pi^{-} \pi^{+}\right)}
\end{aligned}
$$


where $\tau_{B_{d}}$ is the lifetime of $B_{d}$ and the pion mass has been neglected. To be more clear to see the relationship of the CPA with the unparticle phase $\phi_{\mathcal{U}}$, we rewrite the CPA for $\bar{B}_{d} \rightarrow \pi^{+} \pi^{-}$as

$$
\mathcal{A}_{C P}=\frac{-2 \chi_{\pi \pi}^{\mathcal{U}} \sin \alpha \sin \phi_{\mathcal{U}}}{\left|1+\chi_{\pi \pi}^{S M} e^{i \alpha}\right|^{2}+\left|\chi_{\pi \pi}^{\mathcal{U}}\right|^{2}-2 \chi_{\pi \pi}^{\mathcal{H}} \cos \alpha \cos \phi_{\mathcal{U}}}
$$

where $\alpha \equiv \beta+\gamma$ and

$$
\begin{aligned}
\chi_{\pi \pi}^{\mathcal{U}} & =\frac{\tilde{\Delta}_{\mathcal{U}}\left(p^{2}\right)}{4 N_{c} a_{1}} \frac{\left|\xi_{t}^{d}\right|}{\left|\xi_{u}^{d}\right|}\left(C_{L}^{u}+2 r_{\pi} C_{R}^{u}\right), \\
\chi_{\pi \pi}^{S M} & =\frac{1}{a_{1}} \frac{\left|\xi_{t}^{d}\right|}{\left|\xi_{u}^{d}\right|}\left(a_{4}^{u}+2 r_{\pi} a_{6}^{u}\right) .
\end{aligned}
$$

Note that $a_{4(6)}^{q}$ can be derived from Eq. (14) by setting $C_{L(R)}^{q}=0$. Obviously, the CPA in $\bar{B}_{d} \rightarrow \pi^{+} \pi^{-}$depends on not only the weak phase $\alpha$ but also the CP-conserved unparticle phase $\phi_{\mathcal{U}}$.

\section{B. $B \rightarrow \pi K$ decays}

It is known that the decays of $b \rightarrow s q \bar{q}$ are penguin dominant processes as the tree contributions are suppressed by the CKM matrix elements of $V_{u b} V_{u s}^{*}$. Since the unparticle effects are also induced from penguin loops, one expects that they should be significant. In $B \rightarrow \pi K$ decays, there are four specific decay modes. Since the BRs for $B \rightarrow \pi K$ and CPA of $B_{d} \rightarrow \pi^{-} K^{+}$are observed well in experiments, we have to discuss all modes in detail. We begin with our analysis on the decay of $B^{-} \rightarrow \pi^{-} \bar{K}^{0}$. According to the flavor diagram in Fig. 3a, the decay is corresponding to $q=d$. Hence, taking the same conditions as $B_{d} \rightarrow \pi^{-} \pi^{+}$, the decay amplitude for $B^{-} \rightarrow \pi^{-} \bar{K}^{0}$ can be expressed by

$$
\mathcal{M}_{\pi^{-} \bar{K}^{0}}^{\mathcal{U}}=-\frac{G_{F}}{\sqrt{2}} V_{t b} V_{t s}^{*} f_{K} m_{B}^{2} F_{0}^{B \pi}\left(a_{4}^{d \mathcal{U}}+2 r_{K} a_{6}^{d \mathcal{U}}\right),
$$

where $f_{K}$ is the kaon decay constant and $r_{K}=m_{K}^{0} / m_{B}$ with $m_{K}^{0}$ defined as $m_{\pi}^{0}$. Similar to $B^{-} \rightarrow \pi^{-} \bar{K}^{0}$, we can easily find the decay amplitude of $\bar{B}_{d} \rightarrow \pi^{+} K^{-}$by using $q=u$ instead of $q=d$, given by

$$
\mathcal{M}_{\pi^{+} K^{-}}^{\mathcal{U}}=\frac{G_{F}}{\sqrt{2}} f_{K} m_{B}^{2} F_{0}^{B \pi}\left[-V_{t b} V_{t s}^{*}\left(a_{4}^{u \mathcal{u}}+2 r_{K} a_{6}^{u \mathcal{U}}\right)+V_{u b} V_{u s}^{*} a_{1}\right]
$$

where we have included the tree contributions illustrated in Fig. Bk. 


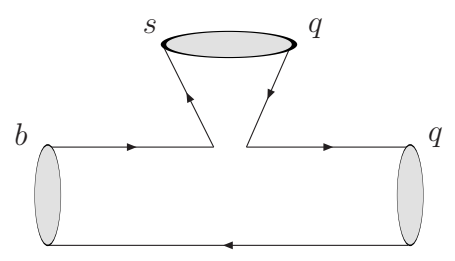

(a)

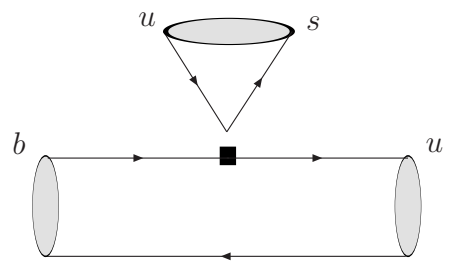

$(c)$

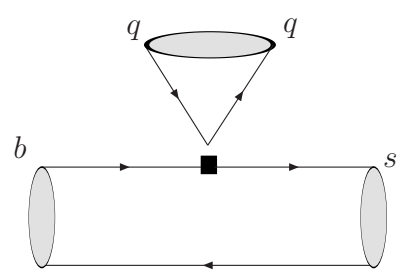

(b)

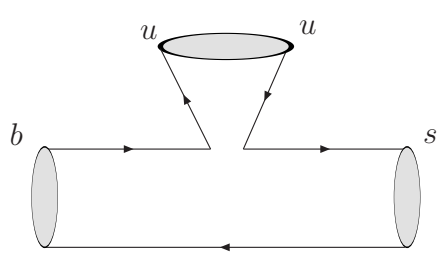

$(d)$

FIG. 3: Flavor diagrams for $B \rightarrow \pi K$ decays dictated by (a) $[(\mathrm{b})]$ penguin and (c) $[(\mathrm{d})]$ tree diagrams.

Next, we analyze the decay of $B_{d} \rightarrow \pi^{0} K^{0}$. Besides the flavor diagrams appearing in the decay of $B^{-} \rightarrow \pi^{-} \bar{K}^{0}$, there are new diagrams shown in Figs. $3 \mathrm{~b}$ and $3 \mathrm{~d}$. In the SM, these contributions correspond to the electroweak penguin and color-suppressed effects, respectively. Taking $q=u$ and $d$ in Figs. 3a and 3b, respectively, the decay amplitude for $\bar{B}_{d} \rightarrow \pi^{0} \bar{K}^{0}$ is given by

$$
\sqrt{2} \mathcal{M}_{\pi^{0} \bar{K}^{0}}^{\mathcal{U}}=\frac{G_{F}}{\sqrt{2}} m_{B}^{2}\left\{V_{t b} V_{t s}^{*}\left[f_{K} F_{0}^{B \pi}\left(a_{4}^{d \mathcal{U}}+2 r_{K} a_{6}^{d \mathcal{U}}\right)-f_{\pi} \zeta F_{0}^{B K}\right]+V_{u b} V_{u s}^{*} f_{\pi} a_{2} F_{0}^{B K}\right\}
$$

where $\zeta=a_{3}^{u \mathcal{U}}-a_{3}^{d \mathcal{U}}+a_{5}^{d \mathcal{U}}-a_{5}^{u \mathcal{U}}$. We note that the new term $f_{\pi} \zeta F_{0}^{B K}$, corresponding to the contribution in Fig. 3b, has opposite in sign to other terms. The reason comes from the flavor wave function of $\pi^{0}$ being $(\bar{u} u-\bar{d} d) / \sqrt{2}$. Note that Fig. 3b picks both components while Fig. 3 a only takes the $\bar{d} d$ component. Since the tree contributions are color suppressed, the corresponding $\mathrm{WC}$ is $a_{2}$.

After introducing the decay amplitudes for $B^{-} \rightarrow \pi^{-} \bar{K}^{0}, \bar{B}_{d} \rightarrow \pi^{+} K^{-}$and $\bar{B}_{d} \rightarrow \pi^{0} \bar{K}^{0}$, the amplitude of $B^{-} \rightarrow \pi^{0} K^{-}$could be immediately obtained as

$$
\begin{aligned}
\sqrt{2} \mathcal{M}_{\pi^{0} K^{-}}^{\mathcal{U}}= & \frac{G_{F}}{\sqrt{2}} m_{B}^{2}\left[V_{t b} V_{t s}^{*}\left(-f_{K} F_{0}^{B \pi}\left(a_{4}^{u \mathcal{U}}+2 r_{K} a_{6}^{u \mathcal{U}}\right)-f_{\pi} \zeta F_{0}^{B K}\right)\right. \\
& \left.+V_{u b} V_{u s}^{*}\left(f_{K} a_{1} F_{0}^{B \pi}+f_{\pi} a_{2} F_{0}^{B K}\right)\right]
\end{aligned}
$$

Clearly, the amplitudes for the first three decay modes all appear in the decay of $B^{+} \rightarrow$ $\pi^{0} K^{+}$. That is, once the first three decays are determined, the decay of $B^{+} \rightarrow \pi^{0} K^{+}$is also fixed. We would point out that although Fig. 3b contributes to the modes of $\pi^{0} \bar{K}^{0}$ and 
$\pi^{0} K^{+}$, similar to the case in $B \rightarrow \pi \pi$, the unparticle effects in this topology will vanish if we take $C_{L(R)}^{d} \approx C_{L(R)}^{u}$. In our study, we also neglect their contributions. In sum, the BRs and CPAs for the $B \rightarrow \pi K$ decays can be found by the definitions in Eq. (17).

\section{Inclusive semileptonic decays of $b \rightarrow q \bar{\ell} \ell$}

If unparticles couple to leptons, we can apply the induced interactions for $b \rightarrow q \mathcal{U}$ to study the semileptonic decays of $b \rightarrow q \bar{\ell} \ell$. The corresponding Feynman diagram is presented in Fig. 4. It is easy to see that due to the CKM suppression, the semileptonic decays with $b \rightarrow d$ are much less than those of $b \rightarrow s$. Hence, in the following discussions, we will concentrate on $b \rightarrow s \ell^{+} \ell^{-}$. Nevertheless, all discussions and formulas could be applied to $b \rightarrow d \ell^{+} \ell^{-}$as well. It is also worth mentioning that because the CKM matrix element $V_{t d}$ carries a CP violating phase, the system of $b \rightarrow d \ell^{+} \ell^{-}$could be even more interesting on $\mathrm{CP}$ violation in the framework of unparticle physics.

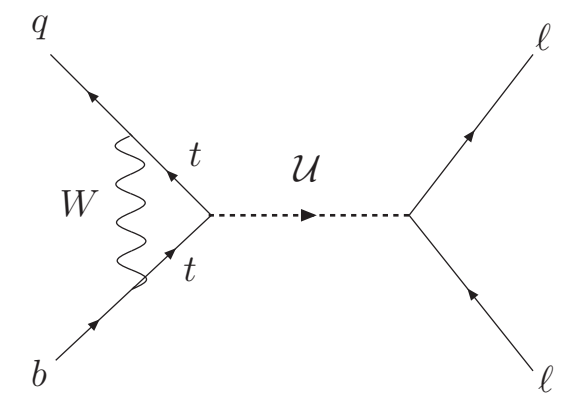

FIG. $4: b \rightarrow q \ell^{+} \ell^{-}$decays induced by unparticle penguin diagram.

Before including new physics interactions into $b \rightarrow s \ell^{+} \ell^{-}$, we write the effective Hamiltonian for the SM as

$$
\mathcal{H}_{\mathrm{eff}}=\frac{G_{F} \alpha_{e m} \lambda_{t}}{\sqrt{2} \pi}\left[H_{1 \mu} L^{\mu}+H_{2 \mu} L^{5 \mu}\right]
$$

with

$$
\begin{aligned}
H_{1 \mu} & =C_{9}^{\mathrm{eff}}(\mu) \bar{s} \gamma_{\mu} P_{L} b-\frac{2 m_{b}}{q^{2}} C_{7}(\mu) \bar{s} i \sigma_{\mu \nu} q^{\nu} P_{R} b \\
H_{2 \mu} & =C_{10} \bar{s} \gamma_{\mu} P_{L} b \\
L^{\mu} & =\bar{\ell} \gamma^{\mu} \ell, \quad L^{5 \mu}=\bar{\ell} \gamma^{\mu} \gamma_{5} \ell
\end{aligned}
$$


where $\alpha_{e m}$ is the fine structure constant, $\lambda_{t}=V_{t b} V_{t s}^{*}, C_{9}^{\text {eff }}$ and $C_{7,10}$ are the Wilson coefficients (WCs) with their explicit expressions given in Ref. 25] for the SM, $m_{b}$ is the current b-quark mass and $q^{2}$ is the invariant mass of the $\ell^{+} \ell^{-}$pair. Although long-distance effects of $c \bar{c}$ bound states could contribute to $C_{9}^{\text {eff }}$, to study the behavior of unparticle physics in the semileptonic decays, for simplicity they are not included in the present study. On the other hand, the bound states could be excluded experimentally by cutting the phase space at the resonant regions. Explicitly, one has that [25]

$$
\begin{aligned}
C_{9}^{\mathrm{eff}}(\mu) & =C_{9}(\mu)+\left(3 C_{1}(\mu)+C_{2}(\mu)\right) h(x, s), \\
h(z, s) & =-\frac{8}{9} \ln \frac{m_{b}}{\mu}-\frac{8}{9} \ln z+\frac{8}{27}+\frac{4}{9} x-\frac{2}{9}(2+x)|1-x|^{1 / 2} \\
& \times\left\{\begin{array}{r}
\ln \left|\frac{\sqrt{1-x}+1}{\sqrt{1-x}-1}\right|-i \pi, \text { for } \mathrm{x} \equiv 4 \mathrm{z}^{2} / \mathrm{s}<1, \\
2 \arctan \frac{1}{\sqrt{x-1}}, \text { for } \mathrm{x} \equiv 4 \mathrm{z}^{2} / \mathrm{s}>1,
\end{array}\right.
\end{aligned}
$$

where $h(z, s)$ describes the one-loop matrix elements of operators $O_{1}^{c}=\bar{s}_{\alpha} \gamma^{\mu} P_{L} b_{\beta} \bar{c}_{\beta} \gamma_{\mu} P_{L} c_{\alpha}$ and $O_{2}^{c}=\bar{s} \gamma^{\mu} P_{L} b \bar{c} \gamma_{\mu} P_{L} c$ [25] with $z=m_{c} / m_{b}$ and $s=q^{2} / m_{b}^{2}$. Comparing to Eq. (8)), we find that the operator structures of the unparticle contributions are the same as those of the SM. The unparticle effects with the SM contributions can be derived by using $C_{9}^{\mathcal{U}}$ and $C_{10}^{\mathcal{U}}$, defined by

$$
\begin{aligned}
& C_{9}^{\mathcal{U}}\left(q^{2}\right)=C_{9}^{\mathrm{eff}}+\frac{\pi}{\alpha_{e m}} \frac{C_{R}^{\ell}+C_{L}^{\ell}}{2} \tilde{\Delta}_{\mathcal{U}}\left(q^{2}\right) e^{-i \phi_{\mathcal{U}}}, \\
& C_{10}^{\mathcal{U}}\left(q^{2}\right)=C_{10}+\frac{\pi}{\alpha_{e m}} \frac{C_{R}^{\ell}-C_{L}^{\ell}}{2} \tilde{\Delta}_{\mathcal{U}}\left(q^{2}\right) e^{-i \phi_{\mathcal{U}}},
\end{aligned}
$$

instead of $C_{9}^{\text {eff }}$ and $C_{10}$, respectively.

With Eq. (24) and the three-body phase space, the inclusive differential decay rate for $b \rightarrow s \ell^{+} \ell^{-}(\ell=e, \mu)$ can be expressed by

$$
\begin{aligned}
\frac{d \Gamma}{d s} & =\frac{G_{F}^{2} m_{b}^{5} \alpha_{\mathrm{em}}^{2}}{768 \pi^{5}}\left|V_{t s} V_{t b}^{*}\right|^{2}(1-s)^{2} R(s), \\
R(s) & =\left(\left|C_{9}^{\mathcal{U}}(s)\right|^{2}+\left|C_{10}^{\mathcal{U}}(s)\right|^{2}\right)(1+2 s)+12 R e\left(C_{7}^{*} C_{9}^{\mathcal{U}}(s)\right)+4\left(1+\frac{2}{s}\right)\left|C_{7}\right|^{2},
\end{aligned}
$$

where the lepton mass has been neglected. Besides the BRs, it has been known that the forward-backward asymmetry (FBA), defined by [31]

$$
\frac{d A_{F B}}{d s}=\frac{\int_{-1}^{1} d \cos \theta d \Gamma / d s d \cos \theta \operatorname{sgn}(\cos \theta)}{\int_{-1}^{1} d \cos \theta d \Gamma / d s d \cos \theta}
$$


could be as a good candidate to probe new physics. By including the unparticle contributions, from Eqs. (28) and (29) we get

$$
\frac{d A_{F B}}{d s}=-3 \frac{s}{R(s)} \operatorname{Re}\left[\left(C_{9}^{\mathcal{U}}(s)+\frac{2}{s} C_{7}\right) C_{10}^{\mathcal{U}^{*}}(s)\right] .
$$

We note that as a whole the decay of $b \rightarrow s \ell^{+} \ell^{-}$is not sensitive to the CP-conserved phases carried by the unparticle propagators. However, it is important to point out that at the low $q^{2}$ regions the unparticle physics has large effects on these physical quantities as the unparticle propagator is proportional to $\left(q^{2}\right)^{d_{\mathcal{U}}-2}$. In these region, we expect that both BR and FBA in $b \rightarrow s \ell^{+} \ell^{-}$have significant deviations from the SM predictions.

\section{NUMERICAL ANALYSIS}

To estimate the numerical values, we take the common parameters to be: $G_{F}=1.166 \times$ $10^{-5} \mathrm{GeV}^{-2}, f_{\pi}=0.13 \mathrm{GeV}, f_{K}=0.16 \mathrm{GeV}, m_{K}^{0}=2.4 \mathrm{GeV}, m_{\pi}^{0}=1.7 \mathrm{GeV}, V_{t d}=$ $8.46 \times 10^{-3} e^{-i \beta}$ with $\beta=25^{\circ}, V_{u b}=3.6 \times 10^{-3} e^{-i \gamma}$ with $\gamma=72^{\circ}$, and $\alpha_{\mathrm{em}}=1 / 129[24]$. For the nonleptonic B decays, since we concentrate on the CPAs, the CP-averaged BRs are regarded as inputs and their world averages are adopted as [32]

$$
\begin{aligned}
\mathcal{B}\left(\bar{B}_{d} \rightarrow \pi^{+} \pi^{-}\right) & =(5.16 \pm 0.22) \times 10^{-6}, \\
\mathcal{B}\left(B^{-} \rightarrow \pi^{-} \bar{K}^{0}\right) & =(23.1 \pm 1.0) \times 10^{-6}, \\
\mathcal{B}\left(\bar{B}_{d} \rightarrow \pi^{+} K^{-}\right) & =(19.4 \pm 0.6) \times 10^{-6} \\
\mathcal{B}\left(\bar{B}_{d} \rightarrow \pi^{0} \bar{K}^{0}\right) & =(10.0 \pm 0.6) \times 10^{-6}, \\
\mathcal{B}\left(B^{-} \rightarrow \pi^{0} K^{-}\right) & =(12.8 \pm 0.6) \times 10^{-6} .
\end{aligned}
$$

For the semileptonic $b \rightarrow s \ell^{+} \ell^{-}$decays, we use the world average as [24]

$$
\mathcal{B}\left(b \rightarrow s \ell^{+} \ell^{-}\right)=(4.5 \pm 1.0) \times 10^{-6}
$$

We first calculate the unparticle contributions to the $\mathrm{BR}$ and $\mathrm{CPA}$ of $\bar{B}_{d} \rightarrow \pi^{+} \pi^{-}$. As mentioned before, to satisfy the indication of the experimental data on $B^{-} \rightarrow \pi^{0} \pi^{-}$, we will require that $C_{L(R)}^{u} \approx C_{L(R)}^{d}=C_{L(R)}^{q}$. Besides the scale dimension $d_{\mathcal{U}}$ and the unparticle scale $\Lambda_{\mathcal{U}}$ fixed to be $1 \mathrm{TeV}$, there are three extra unknown parameters from unparticle physics, i.e. $C_{L}^{t}, C_{L}^{q}$ and $C_{R}^{q}$. Since $C_{L}^{t}$ is always associated with $C_{L(R)}^{q}$, in our numerical calculations, we will use the combined parameters of $\lambda_{L}^{q}=C_{L}^{t} C_{L}^{q}$ and $\lambda_{R}^{q}=C_{L}^{t} C_{R}^{q}$. In addition, we will 
set the available ranges for the variables to be $\left|\lambda_{L(R)}^{q}\right|<0.5$. In terms of Eqs. (15) and (17), the numerical values of the BR and CPA for $\bar{B}_{d} \rightarrow \pi^{-} \pi^{+}$versus the scale dimension $d_{\mathcal{U}}$ are presented in Fig. 5. Because the BR is input, the calculated values are all within $1 \sigma$ errors. Recently, BABAR [33] and BELLE [34] have reported the CPA measurements of

$$
\begin{gathered}
\mathcal{A}_{C P}\left(\bar{B}_{d} \rightarrow \pi^{+} \pi^{-}\right)=(0.21 \pm 0.09 \pm 0.02) \quad(\mathrm{BABAR}) \\
\mathcal{A}_{C P}\left(\bar{B}_{d} \rightarrow \pi^{+} \pi^{-}\right)=(0.55 \pm 0.08 \pm 0.05) \quad(\mathrm{BELLE}),
\end{gathered}
$$

with the average value being

$$
\mathcal{A}_{C P}\left(\bar{B}_{d} \rightarrow \pi^{+} \pi^{-}\right)=0.38 \pm 0.18
$$

According to our results in Fig. 5b, we see that without any QCD phases, the unparticlemediated FCNC with the peculiar CP-conserved phase induced by the penguin diagram could make the CPA of $\bar{B}_{d} \rightarrow \pi^{+} \pi^{-}$as large as 20\%. Clearly, with more and more data accumulated at the $\mathrm{B}$ factories, it is worth to explore whether the unparticle phase is the dominant source for dictating the CPA of $\bar{B} \rightarrow \pi^{+} \pi^{-}$.

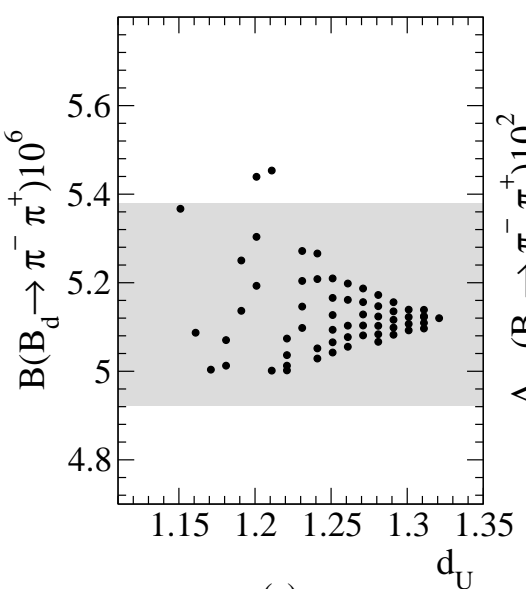

(a)

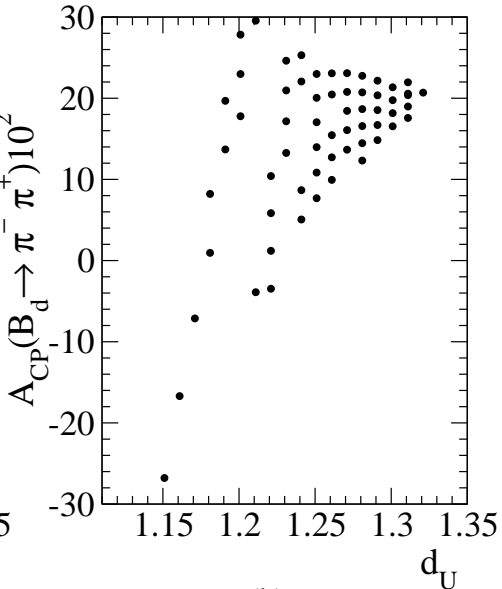

(b)

FIG. 5: (a) BR (in units of $10^{-6}$ ) and (b) CPA (\%) for $B_{d} \rightarrow \pi^{-} \pi^{+}$versus the scale dimension $d_{\mathcal{U}}$ with $\Lambda_{\mathcal{U}}=1 \mathrm{TeV}$ and $\lambda_{L(R)}^{q}<0.5$, where the band in (a) denotes the world average with $1 \sigma$ errors.

With the same set of parameters in $B \rightarrow \pi \pi$, we now study the decays of $B \rightarrow \pi K$. According to the formulas of the decay amplitudes introduced in Eqs. (20), (21), (22) and (23), the values of BRs through unparticle-mediated diagrams are presented in Fig. 6, where 
the band in each figure denotes the world average with $1 \sigma$ errors. It is interesting to see that unparticle physics could make the BRs of $B \rightarrow \pi K$ be consistent with data within $1 \sigma$ world averages. We note that the data of $\mathcal{B}\left(\bar{B}_{d} \rightarrow \pi^{+} \pi^{-}\right)$has been also included to constrain the various unknown parameters.

Although the consistent results in the BRs have been impressive enough, to emphasize the importance of the magic phase in unparticle physics, one should pay attention to the CPA. According to our previous analysis in Eqs. (20) and (22), since the tree contributions are negligible (small) for $B^{-} \rightarrow \pi^{-} \bar{K}\left(\bar{B}_{d} \rightarrow \pi^{0} \bar{K}^{0}\right)$, one can easily understand that the corresponding CPA should be also negligible (small). Nevertheless, we have to remark that the conclusions are correct only for the cases without including final state interactions (FSIs). Note that we have to exclude the discussions on FSIs since we can only control the shortdistance effects. Here, we adopt that the assumption of color transparency dominates the processes in $B$ decays [35]. We conclude that the interesting $\mathrm{CP}$ violating effects in $B \rightarrow \pi K$ decays are the CPAs for $\bar{B}_{d} \rightarrow \pi^{+} K^{-}$and $B^{-} \rightarrow \pi^{0} K^{-}$.
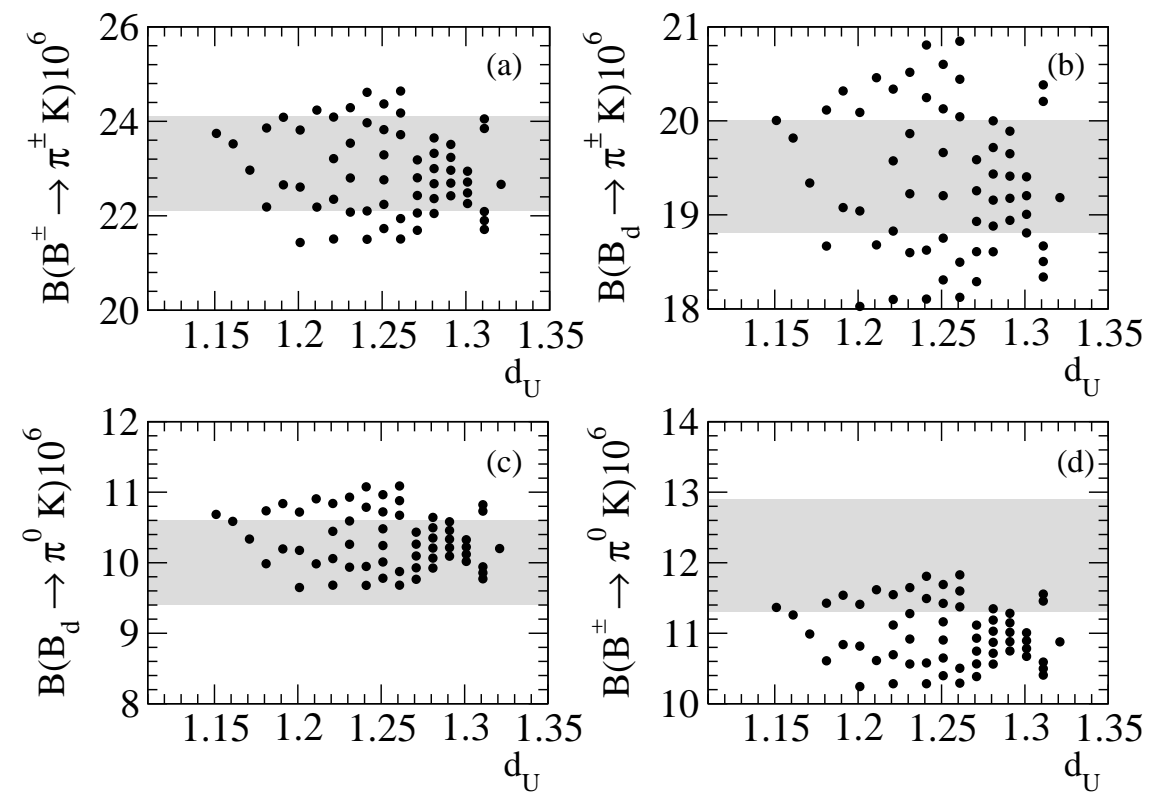

FIG. 6: BRs (in units of $10^{-6}$ ) versus the scale dimension $d_{\mathcal{U}}$ for (a) $B^{-} \rightarrow \pi^{-} \bar{K}^{0}$, (b) $\bar{B}_{d} \rightarrow \pi^{0} \bar{K}^{0}$, (c) $\bar{B}_{d} \rightarrow \pi^{0} \bar{K}^{0}$ and (d) $B^{-} \rightarrow \pi^{0} K^{-}$, where the band in the each figure stands for the world average with $1 \sigma$ errors.

From Eqs. (21) and (23), one finds that the penguin contributions are the same in both decays, the only difference is that there is an extra color-suppressed contribution in $B^{-} \rightarrow$ 
$\pi^{0} K^{-}$. If the color-suppressed $a_{2}$ term in Eq. (23) is dropped, one expects that both modes should have the same CPAs. Therefore, the sign of $a_{2}$ will affect the CPA of $B^{-} \rightarrow \pi^{0} K^{-}$. Using the parameters fitted by the BRs of $B \rightarrow \pi K$, we present the unparticle contributions versus the scale dimension $d_{\mathcal{U}}$ in Fig. 7 , where the circles (squares) dot in each figure denotes $a_{2}=0.14(-0.14)$. We see that the sign of $a_{2}$ has a significant influence on $\mathcal{A}_{C P}\left(B^{-} \rightarrow\right.$ $\left.\pi^{0} K^{-}\right)$. According to the current world average, given by [32]

$$
\begin{gathered}
\mathcal{A}_{C P}\left(\bar{B}_{d} \rightarrow \pi^{+} K^{-}\right)=-0.095 \pm 0.013, \\
\mathcal{A}_{C P}\left(B^{-} \rightarrow \pi^{0} K^{-}\right)=0.047 \pm 0.026,
\end{gathered}
$$

it seems that somewhat different physics exists between the two modes. Plausibly, $a_{2}$ plays an important role in the CPA for $B^{-} \rightarrow \pi^{0} K^{-}$. Since our focus is on the CPA in unparticle physics, further discussions on $a_{2}$ are given elsewhere. The detailed analysis could refer to Ref. [27]. From our results, we see that with the unparticle phase, $\mathcal{A}_{C P}\left(B^{-} \rightarrow \pi^{+} K^{-}\right)$ could be consistent with the current data. As the results in Eq. (35) are not conclusive yet, more precise data are needed to tell whether there is a deviation between $\bar{B}_{d} \rightarrow \pi^{-} K^{+}$and $B^{-} \rightarrow \pi^{0} K^{-}$in the CPAs.

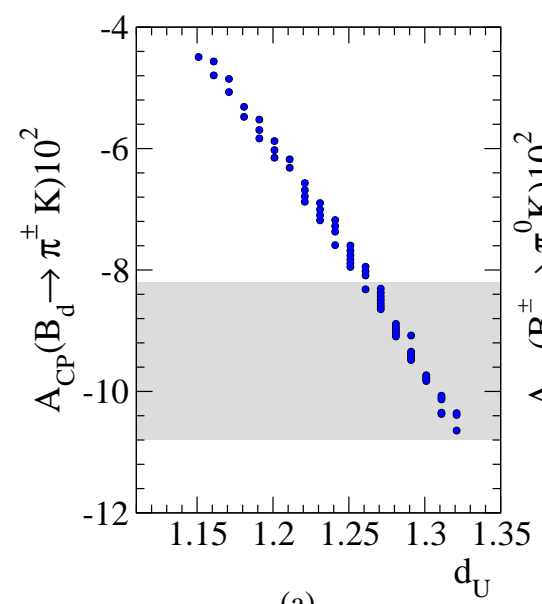

(a)

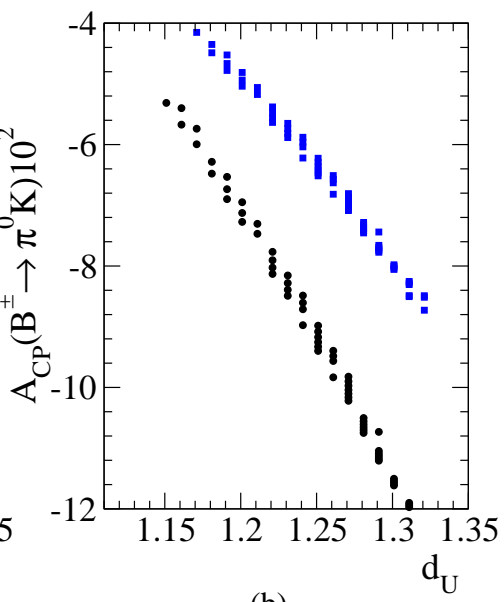

(b)

FIG. 7: CPAs (\%) for (a) $B_{d} \rightarrow \pi^{\mp} K^{ \pm}$and (b) $B^{\mp} \rightarrow \pi^{0} K^{\mp}$ versus the scale dimension $d_{\mathcal{U}}$, where the band in (a) denotes the world average with $1 \sigma$ errors and the circles (squares) dots in each figure represent $a_{2}=0.14(-0.14)$.

Finally, we study the unparticle effects on inclusive semileptonic decays of $b \rightarrow s \ell^{+} \ell^{-}$ with $\ell=e, \mu$. From Eq. (27), one finds that $C_{L}^{t}$ is always associated with $\left(C_{R}^{\ell}+C_{R}^{\ell}\right) / 2$ 
and $\left(C_{R}^{\ell}-C_{L}^{\ell}\right) / 2$. To simplify our numerical analysis, we set $C_{R}^{\ell}=C_{L}^{\ell}$ or $C_{R}^{\ell}=-C_{L}^{\ell}$. We will redefine our parameters to be $C_{L}^{t} C_{L}^{\ell}=C_{L}^{t} C_{R}^{\ell}=\lambda_{V}^{\ell}$ and $C_{L}^{t} C_{L}^{\ell}=-C_{L}^{t} C_{R}^{\ell}=\lambda_{A}^{\ell}$ and discuss the constraints on $\lambda_{V(A)}^{\ell}$. From Eq. (27), we know that the one-loop matrix elements from operators $O_{1}^{c}$ and $O_{2}^{c}$ will generate a CP-conserved QCD phase, which in principle could interfere with the unparticle phase. However, the interference effect between the $C P$-conserved phases of the SM and unparticles is small since the one-loop generated contributions are much smaller than $C_{9} \sim-C_{10} \sim 4$.

Although $b \rightarrow s \ell^{+} \ell^{-}$cannot be the candidate to probe the unique unparticle phase, we can utilize the decays to give strong constraints on the unparticle couplings to leptons, i.e. $\lambda_{V(A)}^{\ell}$. In terms of Eq. (28) and the values for the common parameters, the unparticle contributions to the BRs of $b \rightarrow s \ell^{+} \ell^{-}$are presented in Fig. 8, where (a)[(b)] denotes the contributions of $\lambda_{V[A]}^{\ell}$, the horizontal thin lines are the SM contributions and the thick solid, dashed and dash-dotted lines correspond to $\lambda_{V[A]}^{\ell}=0.005,0.01$ and 0.05, respectively. The bands in the diagrams are the world average with $1 \sigma$ errors. From the figure, we see clearly that with a specific value for $\lambda_{V(A)}^{\ell}$, the $\mathrm{BR}$ of $b \rightarrow s \ell^{+} \ell^{-}$is very sensitive to the scale dimension $d_{\mathcal{U}}$. To understand the sensitivity, we need to examine the behavior of the

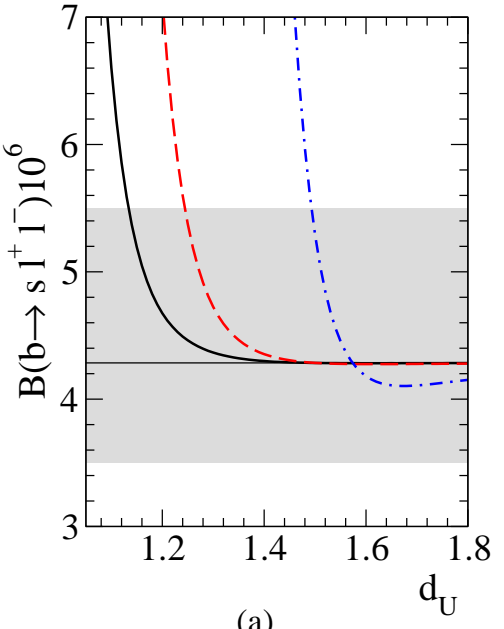

(a)

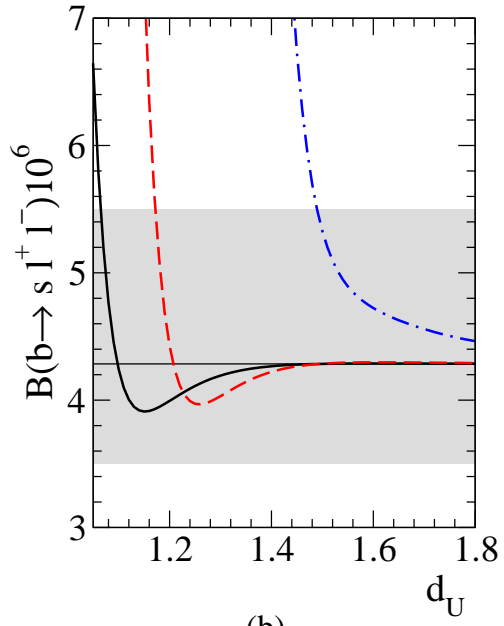

(b)

FIG. 8: $\mathrm{BR}$ ( in units of $10^{-6}$ ) for $b \rightarrow s \ell^{+} \ell^{-}$with (a) $C_{L}^{t} C_{R}^{\ell}=C_{L}^{t} C_{L}^{\ell}=\lambda_{V}^{\ell}$ and (b) $C_{L}^{t} C_{R}^{\ell}=$ $-C_{L}^{t} C_{L}^{\ell}=\lambda_{A}^{\ell}$ versus the scale dimension $d_{\mathcal{U}}$, where the thick solid, dashed and dash-dotted lines correspond to $\lambda_{V(A)}^{\ell}=0.005,0.01$ and 0.05 , respectively, while the horizontal lines are the SM contributions and the bands denote the world average with $1 \sigma$ errors.

unparticle propagator and the unparticle couplings to fermions. With Eqs. (2) and (5), we 
know that the $q^{2}$-dependence in the BR will behave like

$$
\left[\frac{1}{q^{2}}\left(\frac{q^{2}}{\Lambda_{\mathcal{U}}^{2}}\right)^{d_{\mathcal{U}}-1}\right]^{2} .
$$

It is clear that for the three-body $b \rightarrow s \ell^{+} \ell^{-}$decays, the enhancement of unparticle effects is at the small invariant mass of $\ell^{+} \ell^{-}$. To be more clear, we display the differential BRs for
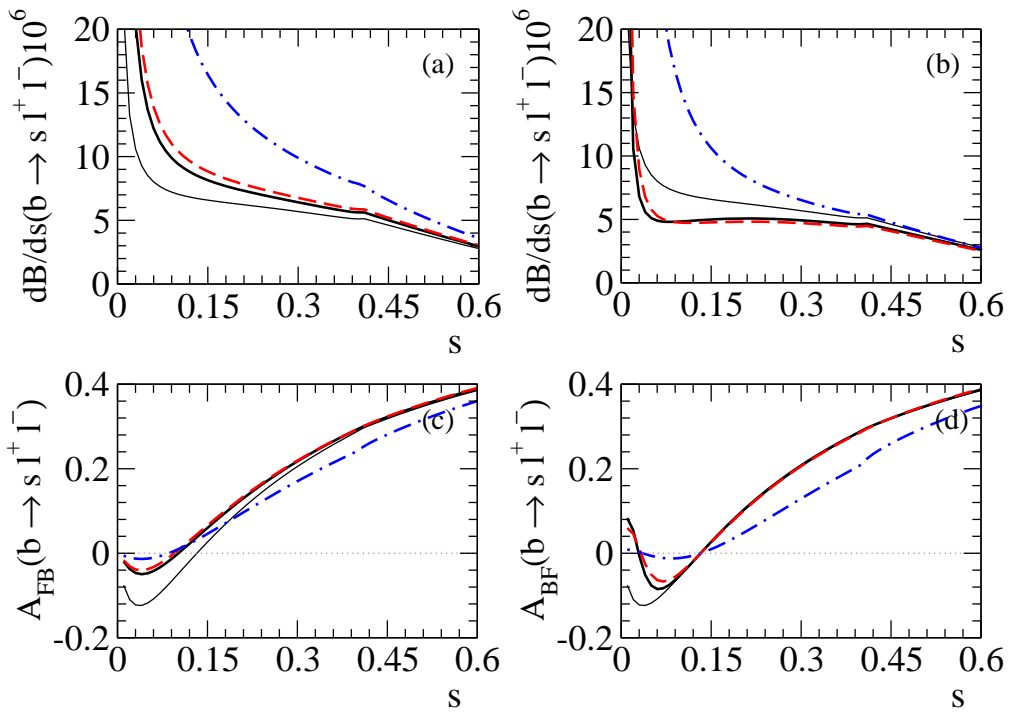

FIG. 9: (a)[(b)] Differential BR ( in units of $10^{-6}$ ) and (c) $[(d)]$ FBA for $b \rightarrow s \ell^{+} \ell^{-}$as functions of $s=q^{2} / m_{b}^{2}$, where the thick solid, dashed and dash-dotted lines correspond to $d_{\mathcal{U}}\left(\lambda_{V[A]}^{\ell}\right)=$ 1.1(0.005), 1.2(0.01) and 1.4(0.05), respectively, while the thin lines are the SM contributions.

$b \rightarrow s \ell^{+} \ell^{-}$as functions of $s=q^{2} / m_{b}^{2}$ in Figs. 9a and 9b, where the solid, dashed and dashdotted lines stand for $d_{\mathcal{U}}\left[\lambda_{V(A)}^{\ell}\right]=1.1[0.005], 1.2[0.01]$ and $1.4[0.05]$. The large deviation at the small $s$ region could confirm our argument.

For the FBA in $b \rightarrow s \ell^{+} \ell^{-}$, from Eq. (30), the numerical values of the unparticle contributions as functions of the invariant mass $s$ are shown in Figs. 9r and 9d. Clearly, the FBA at the small $s$ region is also sensitive to the unparticle physics. In addition, one observes that the nonvanished $\lambda_{V}^{\ell}$ associated with $C_{9}^{\mathcal{U}}$ could shift the zero point of the FBA to be lower. However, the nonvanished $\lambda_{A}^{\ell}$ associated with $C_{10}^{\mathcal{U}}$ cannot change the zero-crossing point. The reason could be understood from Eq. (30), where the zero point can only happen at $C_{9}^{\mathcal{U}}+2 C_{7} / s=0$. 


\section{CONCLUSIONS}

We have studied the implications of the $\mathrm{CP}$ conserving phases in the unparticle propagators. We have demonstrated that these peculiar phases have an important impact on $\mathrm{CP}$ violation since they could act as the strong phases needed to induce the direct $\mathrm{CP}$ asymmetry. Without including the QCD phases, we have examined the unparticle phase effects on the direct $\mathrm{CP}$ asymmetries in the exclusive $\bar{B}_{d} \rightarrow \pi^{+} \pi^{-}$and $B \rightarrow \pi K$ decays, in which FCNCs are forbidden at tree level but induced by one-loop unparticle penguin diagrams. We have obtained interesting and consistent results comparing to the experimental data. Moreover, we have found that the unparticle effects will significantly enhance the differential branching ratio of $b \rightarrow s \ell^{+} \ell^{-}$at the small invariant mass of $\ell^{+} \ell^{-}$so that the

couplings of unparticles to leptons suffer strong constraints. The forward-backward asymmetries for the decays of $b \rightarrow s \ell^{+} \ell^{-}$due to the unparticle effects have also been investigated.

\section{Acknowledgments}

This work is supported in part by the National Science Council of R.O.C. under Grant \#s: NSC-95-2112-M-006-013-MY2 and NSC-95-2112-M-007-059-MY3.

[1] H. Georgi, Phys. Rev. Lett. 98, 221601 (2007) arXiv:hep-ph/0703260.

[2] H. Georgi, Phys. Lett. B650, 275 (2007) [arXiv:0704.2457].

[3] K. Cheung, W. Y. Keung and T. C. Yuan, Phys. Rev. Lett. 99, 051803 (2007) arXiv:0704.2588.

[4] M. Luo and G. Zhu, arXiv:0704.3532 [hep-ph].

[5] C. H. Chen and C. Q. Geng, arXiv:0705.0689 [hep-ph].

[6] G. J. Ding and M. L. Yan, arXiv:0705.0794 [hep-ph].

[7] Y. Liao, arXiv:0705.0837 [hep-ph].

[8] T. M. Aliev, A. S. Cornell and N. Gaur, arXiv:0705.1326 [hep-ph].

[9] S. Catterall and F. Sannino, arXiv:0705.1664 [hep-lat].

[10] X. Q. Li and Z. T. Wei, arXiv:0705.1821 [hep-ph].

[11] C. D. Lu, W. Wang and Y. M. Wang, arXiv:0705.2909 [hep-ph]. 
[12] M. A. Stephanov, arXiv:0705.3049 [hep-ph].

[13] P. J. Fox, A. Rajaraman and Y. Shirman, arXiv:0705.3092 [hep-ph].

[14] N. Greiner, arXiv:0705.3518 [hep-ph].

[15] H. Davoudiasl, arXiv:0705.3636 [hep-ph].

[16] D. Choudhury, D. K. Ghosh and Mamta, arXiv:0705.3637 [hep-ph].

[17] S. L. Chen and X. G. He, arXiv:0705.3946 [hep-ph].

[18] T. M. Aliev, A. S. Cornell and N. Gaur, arXiv:0705.4542 [hep-ph].

[19] P. Mathews and V. Ravindran, arXiv:0705.4599 [hep-ph].

[20] S. Zhou, arXiv:0706.0302 [hep-ph].

[21] G. J. Ding and M.L. Yan, arXiv:0706.0325 [hep-ph].

[22] N. Cabibbo, Phys. Rev. Lett. 10, 531 (1963); M. Kobayashi and T. Maskawa, Prog. Theor. Phys. 49, 652 (1973).

[23] L. Wolfenstein, Phys. Rev. Lett. 51, 1945 (1983).

[24] Particle Data Group, W.M. Yao et al., J. Phys. G: Nucl. Part. Phys. 33, 1 (2006).

[25] G. Buchalla, A. J. Buras and M. E. Lautenbacher, Rev. Mod. Phys 68, 1125 (1996) arXiv:hep-ph/9512380.

[26] M. Beneke and M. Neubert, Nucl.Phys. B675, 333 (2003).

[27] H.N. Li and S. Mishima, Phys. Rev. D73, 114014 (2006).

[28] C.H. Chen, arXiv:hep-ph/0601019.

[29] C.M. Arnesen, I.Z. Rothstein and I.W. Stewart, arXive:hep-ph/0611356.

[30] C.H. Chen et al., Phys. Rev. D72, 054011 (2005); C.H. Chen and C.Q. Geng, Phys. Rev. D75, 054010 (2007).

[31] A. Ali, T. Mannel and T. Morozumi, Phys. Lett. B273, 505 (1991).

[32] Heavy Flavor Averaging Group, E. Barberioet al., arXiv:0704.3575 [hep-ex]; online update at http://www.slac.stanford.edu/xorg/hfag.

[33] J. Biesiada [BABAR Collaboration], Proceedings to the Lake Louise Winter Institute 2007, arXiv:0705.1001 [hep-ex].

[34] H. Ishino et al. [BELLE Collaboration], arXive:hep-ex/0608035.

[35] J. D. Bjorken, Nucl. Phys. Proc. Suppl. 11, 325 (1989). 